\title{
Expression of PAX-5 in B Cell Hodgkin and Non Hodgkin Lymphoma
}

\author{
Muhammad Rahil Khan*, Arsalan Ahmad, Naila Kayani, Khurram Minhas
}

\begin{abstract}
Background: B-cell malignancies including Precursor B-cell lymphoblastic lymphoma/leukemia and Hodgkin Lymphoma show a wide spectrum of B-cell differentiation from early stage B-cell precursors to mature B-cells ending in terminal differentiation to plasma cells. Pan-B-cell antigens routinely used for the diagnosis of B-cell lymphoma, include CD19, CD20, CD22 and CD79a.PAX-5 protein, also known as B-cell-specific activation protein is a B-cell-specific transcription factor; essential for commitment and functional maintenance used in the diagnosis of B cell Hodgkin and non-Hodgkin lymphoma. PAX-5 show nuclear positivity in B cell lymphomas and moderate (dim) positivity in Hodgkinlymphoma Reed Sternberg cells make this marker ideal for diagnosing B cell malignances. Objective: To determine the expression of PAX-5 in B cell Hodgkin and non-Hodgkin Lymphoma in order to improve the diagnosis of B-cell lymphomas. Methods: In this Prospective study, all the cases of B cell lymphoma diagnosed at The Aga Khan University Hospital, Karachi from July 2010 to July 2011were included. A panel of Immunohistochemical stain was performed in all cases along with additional PAX- 5 stain with appropriate controls. Results: Total 125 cases were included. Hodgkin Lymphoma (Mixed cellularity) was the commonest B-cell lymphoma subtype, 32 (25\%) cases. Other common subtypes included Hodgkin lymphoma (Nodular sclerosis subtype), diffuse large B-cell lymphoma and B lymphoblastic lymphoma. Conclusion: This study demonstrates that PAX-5 is the most sensitive and reliable immuhohistochemicalmarker in the diagnosis of B cell Hodgkin and non-Hodgkin lymphoma.
\end{abstract}

Keywords: PAX-5- Hodgkin Lymphoma- Non Hodgkin Lymphoma

Asian Pac J Cancer Prev, 19 (12), 3463-3466

\section{Introduction}

Mature B-cell neoplasms comprise $>90 \%$ of all lymphoid neoplasms along with precursor B lymphoblastic lymphoma/leukemia and classic Hodgkin lymphoma (Dong et al., 2008; Swerdlowet al., 2010) These neoplasms comprise of neoplastic B cells at a wide range of differentiation, from precursor $B$ cells in the B-cell maturation pathway with only partial expression of pan-B-cell antigens to late-stage B cells undergoing plasma cell differentiation with someloss of B-cell signatures (Dong et al., 2008). Classic Hodgkin lymphoma, traditionally considered a separate entity is now considered as a neoplasm of B cell lineage which creates a greater overlap between classic Hodgkin lymphoma and many forms of B cell malignancies (Swerdlowet al., 2010; Barakzai et al., 2009; Sultan et al., 2016). Recognition of the B-cell lineage is crucial for diagnosis and classification of these lymphomas which is greatly dependent on immunohistochemistry (Jensenetal., 2007).

There have been a few pan-B-cell antigens routinely used for the diagnosis of B-cell lymphoma, such as CD20 and CD79a (Aftab et al., 2006). Precursor B-lymphoblastic leukaemia /lymphoma often lack CD20 expression. On the other hand, it has been well documented that CD20 and CD79a may be expressed in subsets of T-cell malignancies (Dong et al., 2008).

PAX-5 is a member of the paired box domain gene family that encodes nuclear transcription factors important in development, differentiation, cell migration and proliferation. PAX-5 protein is expressed as a nuclear marker in cells of B-lineage that spans the differentiation spectrum and is found to be expressed in pre- and mature B cells, but not in plasma cells (McCuneet al., 2006). PAX-5 is also expressed by Reed-Sternberg cells in classic Hodgkin lymphoma where the specific weak nuclear immuno-reactivity of PAX-5 effectively distinguishes Reed-Sternberg cells from other reactive components, such as polykaryocytes, histiocytes, dendritic cells and endothelial cells (Dong et al., 2008). PAX-5 is a good and reliable immunomarker in diagnosing $\mathrm{B}$ cell non Hodgkin lymphomas and Hodgkin lymphomas (Mhawech et al., 2007).

PAX-5 is the most sensitive and reliable 
immunohistochemical marker for B-cell malignancies (Dong et al., 2008). The nuclear positivity of PAX-5 makes it different from other pan B cells markers (CD20 and CD79a) which are membrane markers (Dong et al., 2008). PAX-5 is expressed in the absence of expression of other pan-B-cell markers, suggesting that the inclusion of PAX-5 in a panel of antibodies to diagnose B cell lymphomas would be very supportive (Jensen et al., 2007). PAX-5 has not been found in mature normal and malignant $\mathrm{T} /$ natural killer cells (Dong et al., 2008). The purpose of this study is to determine PAX-5 expression in B-cell lymphomas through immunohistochemistry in order to improve the diagnosis of B cell lymphomas.

\section{Materials and Methods}

\section{Study Settings}

The study was conducted in the section of Histopathology, Department of Pathology and Laboratory Medicine of The Aga Khan University Hospital, Karachi. The section receives over seventy thousand surgical specimens per year. These cases include biopsies from inpatients and outside referrals from all over Pakistan. This cross sectional study was conducted over a period of one year (July 2010 to July 2011). Non probability consecutive sampling was done to include all the cases of B cell lymphoma (Hodgkin and non Hodgkin lymphoma) diagnosed over a period of one year.

\section{Data Collection}

Study variables including, age, gender, site, immunohistochemical stains and diagnosis were recorded on a predesigned proforma. The morphology was assessed microscopically by two pathologists (consultant and resident). For each case, one representative section was selected and immunohistochemical stains were performed. These included Leucocyte common antigen (LCA/CD45), Pan B (CD20, CD79a), Pan T (CD3), CD30 (ki-1), CD15 (GAA), CD 5, CD10, BCL2, cyclin D1 and PAX-5 immunohistochemical stains.For PAX-5, nuclear brown staining of B cell was regarded as positive and it was scored as 0 (weak), 1 (moderate), and 2 (strong) on the basis of intensity of staining (McCuneet al., 2006; Johri et al., 2016). A diagnosis of Hodgkin lymphoma was given for those cases in which typical Reed Sternberg (RS) cells were present in a mixed background comprising of lymphocytes, eosinophils and plasma cells. These cases demonstrated positivity for CD15, CD30 and PAX-5 in the RS cells and were negative for LCA immunostain. The diagnosis of Non Hodgkin lymphoma was given for those cases which showed positivity for Pan B (CD20, CD79a), CD5, CD10, BCL2, cyclin D1, LCA and PAX-5.

\section{Results}

A total of 125 cases were diagnosed as B cell lymphoma (Hodgkin and non Hodgkin) during the study period. There were 87 males $(69.60 \%)$ and 38 females (30.40\%) with male to female ratio of 2.3:1. The mean age was $35 \pm 20.64$ years (range from $3-83$ years). Out of 125 cases, $86(68.80 \%)$ samples were from lymph nodes while $39(31.20 \%)$ were from extra-nodal (sites other than lymph nodes). All the included cases in the study showed positivity for pax-5 (100\%) out of which $64(51.2 \%)$ cases expressed moderate (dim) positivity whereas, 61 $(48.8 \%)$ cases showed strong positivity. No differences with respect to the site of lymphoma (Nodal and extra nodal), age or gender of patients were observed. Hodgkin Lymphoma (Mixed cellularity) was the commonest B-cell lymphoma subtype detected in $32(25.6 \%)$ cases. Other common subtypes included Hodgkin lymphoma (Nodular sclerosis subtype), diffuse large B-cell lymphoma and B lymphoblastic lymphoma (Table-1). The PAX-5 showed strong positivity in background $\mathrm{B}$ cells and moderate (dim) positivity in Hodgkin lymphoma RS cells. B-cell Non-hodgkin lymphomas are strongly positive for PAX-5 and only two cases showed moderate (dim) positivity.

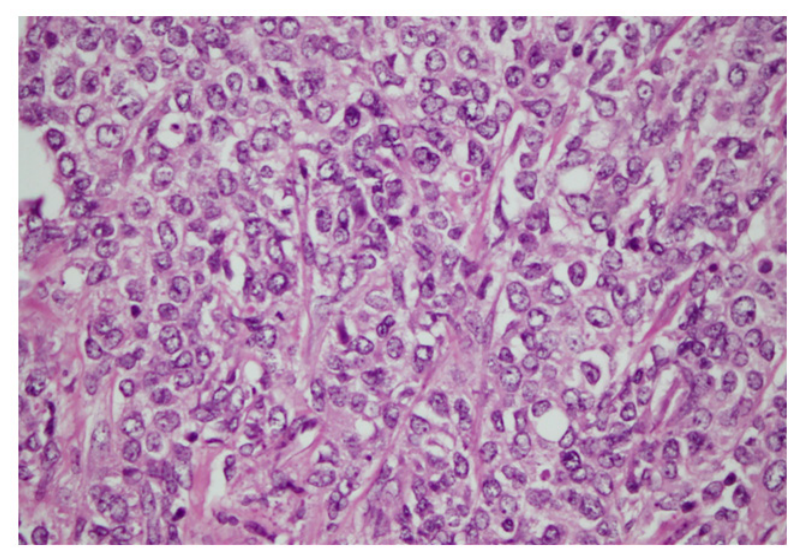

Figure 1. Diffuse Large B Cell Non Hodgkin Lymphoma. (H\&E 40X)

Table 1. Pax-5 Positivity in Various Subtypes of B-cell Lymphomas

\begin{tabular}{|c|c|c|c|}
\hline \multirow[t]{3}{*}{ Lymphoma Subtype } & \multicolumn{2}{|c|}{ PAX-5 } & \multirow[t]{3}{*}{ Total } \\
\hline & \multicolumn{2}{|c|}{ Number of cases $(\%)$} & \\
\hline & Moderate & Strong & \\
\hline DLBCL* & $1(1.6 \%)$ & $26(42.6 \%)$ & $27(21.6 \%)$ \\
\hline Burkitt Lymphoma & $0(0 \%)$ & $4(6.6 \%)$ & $4(3.2 \%)$ \\
\hline $\begin{array}{l}\text { Tcell Rich B Cell } \\
\text { Lymphoma }\end{array}$ & $0(0 \%)$ & $4(6.6 \%)$ & $4(3.2 \%)$ \\
\hline CLL/SLL* & $0(0 \%)$ & $6(9.8 \%)$ & $6(4.8 \%)$ \\
\hline $\begin{array}{l}\text { Marginal Zone B Cell } \\
\text { Lymphoma }\end{array}$ & $0(0 \%)$ & $4(6.6 \%)$ & $4(3.2 \%)$ \\
\hline $\begin{array}{l}\text { Intermediate Between } \\
\text { DLBCL and Hodgkin } \\
\text { Lymphoma }\end{array}$ & $1(1.6 \%)$ & $1(1.6 \%)$ & $2(1.6 \%)$ \\
\hline B Lymphoblastic Lymphoma & $0(0 \%)$ & $16(26.2 \%)$ & $16(12.8 \%)$ \\
\hline $\begin{array}{l}\text { Hl Nodular Sclerosis } \\
\text { Subtype }\end{array}$ & $28(43.8 \%)$ & $0(0 \%)$ & $28(22.4 \%)$ \\
\hline $\begin{array}{l}\text { H1 Mixed } \\
\text { Cellularitysubtype }\end{array}$ & $32(50 \%)$ & $0(0 \%)$ & $32(25.6 \%)$ \\
\hline $\begin{array}{l}\text { Nodular Lymphocyte } \\
\text { Predominant } \mathrm{Hl}\end{array}$ & $1(1.6 \%)$ & $0(0 \%)$ & $1(0.8 \%)$ \\
\hline IPSID* & $1(1.6 \%)$ & $0(0 \%)$ & $1(0.8 \%)$ \\
\hline & $64(100 \%)$ & $61(100 \%)$ & $125(100 \%)$ \\
\hline
\end{tabular}

*HL Hodgkin lymphoma, DLBCL Diffuse large B cell lymphoma, CLL/SLL Chronic lymphocytic leukemia and small lyphocytic lymphoma and IPSID Immunproliferative small intestinal disease. 


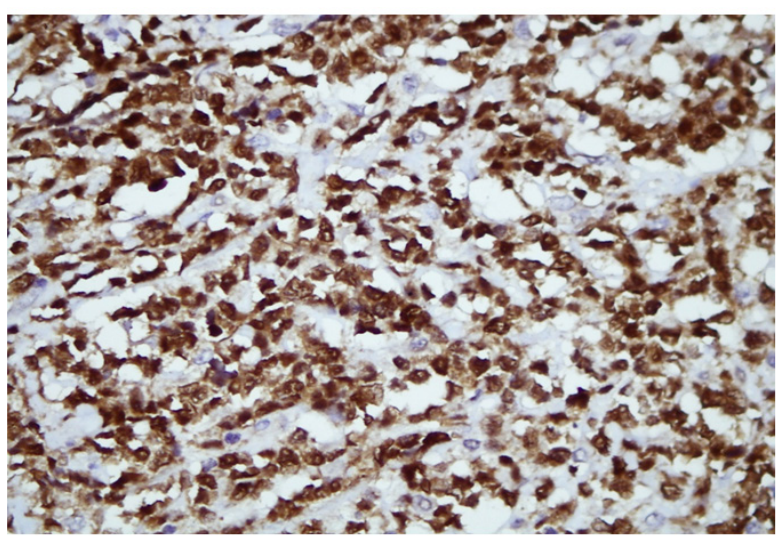

Figure 2. Diffuse Large B Cell Non Hodgkin Lymphoma Showing Strong Nuclear Positivity of PAX-5.

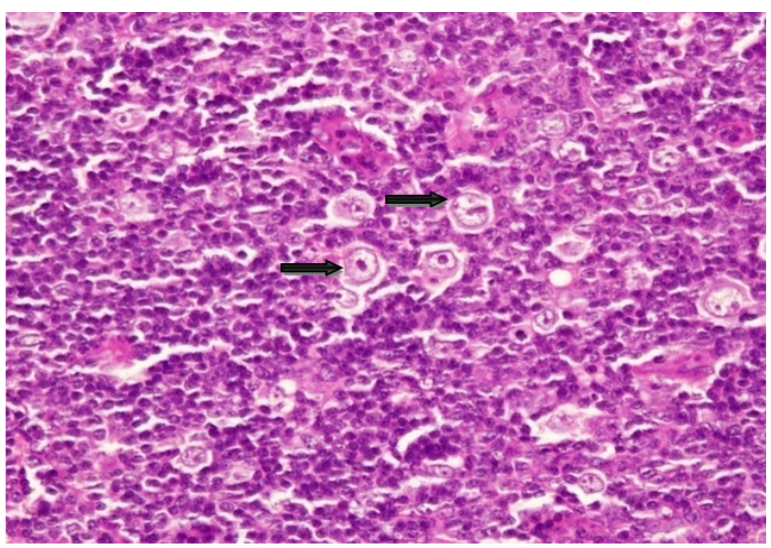

Figure 3. Reed-Sternberg Cells are Scattered Against a Mixed Infiltrate. (H\&E 40X). Marked with

Figures 1-4 show patterns of PAX-5 expression in various B-cell lymphomas.

\section{Discussion}

The transcription factors e2a, ebf1 and PAX-5 cause common lymphoid progenitors to develop into B-cells (Sigvardsson et al., 2002). The absence of any one of these transcription factors block B-cell development at an early stage. Pax-5 expression is mostly limited to cells of B-lymphocyte origin, and Pax-5 has been shown to play an essential role in B-lymphoid lineage commitment. PAX-5 gene expression is activated during the pro-B-cell stage of development and continues through the pre-B-cell and mature B-cell stages, but is absent in normal plasma cells (Zwollo et al.,1997; Sigvardsson et al., 2002; Dong et al., 2008; Adams et al., 1992; Hangman et al 2000).

The nuclear positivity of PAX-5 makes it different from other pan B cells markers (CD20 and CD79a) which are membrane markers (Dong et al., 2008). PAX-5 is expressed in all B cell lymphoma as well as in neoplastic cells of classic Hodgkin lymphoma (Buettner et al., 2005). Although CD20 and CD79a are most commonly used to demonstrate B-cell lineage in B-cell Non-Hodgkin lymphomas, but they have their limitations. CD20 is expressed from late precursor B-cells through mature

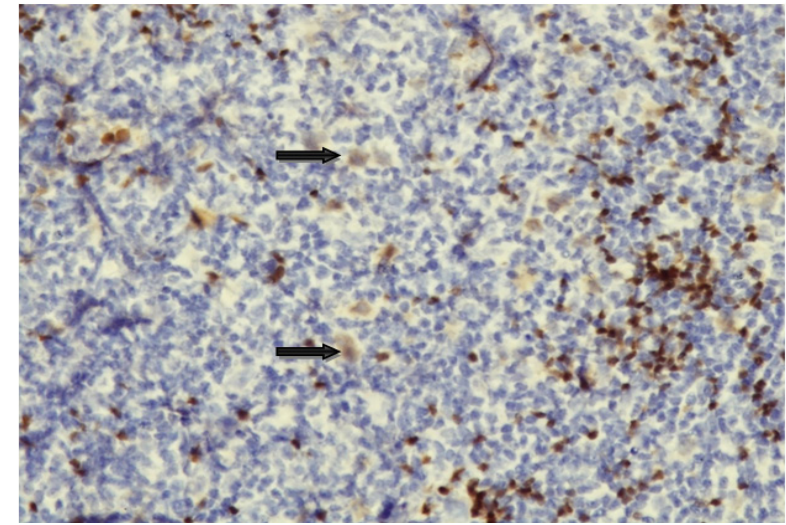

Figure 4.Reed-Sternberg Cells Show Moderate (dim) Positivity for PAX-5 Immunohistochemical Stain Marked with $\Longrightarrow$, which is helpful for histopathologists to identify reed-sternberg cells while background B cell are strongly positive for PAX-5.

B-cells and therefore may not be expressed in Precursor B Acute lymphoblastic lymphoma/leukemia. Likewise CD20 expression may be lost in B-cell lymphomas status post Rituximab treatment and may also be negative or weakly expressed in some B-cell lymphomas. CD79a is expressed in all stages of B-cell differentiation including normal plasma cells therefore CD79a cannot be used to differentiate between B-cells and plasma cells. CD79a can also be aberrantly expressed in precursor $\mathrm{T}$ Acute lympoblastic lymphomas and Acute myeloid leukemias. In addition CD 79a is also not expressed in neoplastic cells of classic Hodgkin lymphoma which are now known as of B cell origin. PAX- 5 has emerged as a marker which is expressed in precursor B cells and also in neoplastic cells of classic Hodgkin lymphoma (Dong et al., 2008; Swerdlowet al., 2010). These properties make it decisive marker than CD 20 and CD 79a in difficult cases. Another maker CD19 is most specific and is found in the broadest spectrum of B cells but in most laboratories it can only be analyzed by flow cytometry with fresh cells (Dong et al., 2008; Masir et al., 2006). B cell lymphoma, status post rituximab lacks CD 20 expression, however strong expression of PAX-5 makes it an ideal immunohistochemical marker for establishing the diagnosis in such cases (Chu et al., 2006)

In one of the study of Dong et al PAX-5 expression was identified in $97 \%$ cases of B-lymphoproliferative disorders (Hodgkin and Non Hodgkin Lymphomas) (Dong et al., 2008). In another study of Johri et al PAX-5 expression was identified in 23/24(95.8\%) in hodgkin lymphoma and 32/39 in B cell non hodgkin lymphoma (Johri et al., 2016). In comparison to other studies our study all cases are PAX-5 positive, all Hodgkin lymphoma cases showed moderate(dim) positivity in RS cells and Non-hodgkin lymphomas are strongly positive for PAX-5 and only two cases showed moderate(dim)positivity instead of strong positivity.

PAX-5 expression is seen in all B cell lymphoma and Hodgkin lymphoma (Buettner et al., 2005). PAX-5 helps to differentiate Hodgkin lymphoma, anaplastic large cell lymphoma and $\mathrm{T}$ cell rich $\mathrm{B}$ cell lymphoma. PAX-5 
expression is seen in Hodgkin lymphoma and T cell rich B cell lymphoma (Shahabuddin et al., 2003; Dong et al., 2008). However, anaplastic large cell lymphomas are negative.

PAX-5 is negative in plasma cell neoplasms and T cell lymphoproliferative disorders, hence it is useful in excluding the two disorders. The lack of PAX-5 expression in most CD30-positive non-hematopoietic malignancies (embryonal carcinoma and Seminoma) and T-cell lymphomas, such as anaplastic large cell lymphoma (ALCL), suggest that the absence of PAX-5 may be used to confirm non-B-cell lineage. No evidence of PAX-5 expression is seen in medulloblastoma, neuroblastoma, astrocytomas and bladder tumor by immunohistochemistry and in many other epithelial and mesenchymal tumors (Desouki et al., 2010; Mhawech et al., 2007).

In conclusion, this study demonstrates that Pax-5 is consistently expressed in all the cases of B cell lymphoma (Hodgkin and non Hodgkin). Therefore, it should be included in diagnostic panel of all suspected B-cell malignancies. Moderate (dim) Pax-5 positivity in Reed Sternberg cells is particularly helpful in differentiating Hodgkin from non Hodgkin B cell lymphomas.

\section{Acknowledgments}

The authors would like to thanks Dr Muhammad Shariq Shaikh for his help in this research. The study is a student thesis; the proposal wasapproved by College of Physicians and Surgeon Pakistan (CPSP).

\section{References}

Adams B, Dörfler P, Aguzzi A, et al (1992). Pax-5 encodes the transcription factor BSAP and is expressed in B lymphocytes, the developing CNS, and adult testis. Genes Dev, 6, 1589-607

Aftab K, Bhurgri Y, Pervez S (2006). Small B cell Non-Hodgkins Lymphoma in Pakistan. J Pak Med Assoc, 56, 22-5.

Barakzai MA, Pervez S (2009). CD20 positivity in classical Hodgkin's lymphoma: Diagnostic challenge or targeting opportunity. Indian J Pathol Microbiol, 52, 6-9.

Buettner M, Greiner A, Avramidou A, Jäck HM, NiedobitekG (2005). Evidence of abortive plasma cell differentiation in Hodgkin and Reed-Sternberg cells of classical Hodgkin lymphoma. Hematol Oncol, 23, 127-32.

Chu PG, Loera S, Huang Q, Weiss LM (2006). Lineage determination of CD20-B-Cell neoplasms: an immunohistochemical study. Am J ClinPathol, 126, 534-44.

Desouki MM, Post GR, Cherry D, LazarchickJ (2010). PAX-5: a valuable immunohistochemical marker in the differential diagnosis of lymphoid neoplasms. Clin Med Res, 8, 84-8.

Dong HY, Browne P, Liu Z, GangiM (2008). PAX-5 is invariably expressed in B-cell lymphomas without plasma cell differentiation. Histopathology, 53, 278-87.

Hangman J, Wheat W, Fitzsimmons D, et-al (2000). Pax-5/BSAP: regulator of specific gene expression and differentiation in B lymphocytes. Curr Top MicrobiolImmunol, 245, 169-94.

Jensen KC, Higgins JPT, Montgomery K, et al (2007). The utility of PAX5 immunohistochemistry in the diagnosis of undifferentiated malignant neoplasms. Mod Pathol, 20, 871-7.

Johri N, Patne SC, Tewari M, Kumar M (2016). Diagnostic utility of PAX5 in Hodgkin and Non-Hodgkin Lymphoma: A study from Northern India. J Clin Diagn Res, 10, XC04-07.

Masir N, Marafioti T, Jones M, Natkunam Y, et al (2006). Loss of CD19 expression in B-cell neoplasms. Histopathology, 48, 239-46

McCune RC, Syrbu SI, Vasef MA (2006). Expression profiling of transcription factors Pax-5, Oct-1, Oct-2, BOB.1, and PU.1 in Hodgkin's and non-Hodgkin's lymphomas: a comparative study using high throughput tissue microarrays. Mod Pathol, 19, 1010-8.

Mhawech-Fauceglia P, Saxena R, Zhang S, et al (2007). Pax-5 immunoexpression in various types of benign and malignant tumours: a high-throughput tissue microarray analysis. J Clin Pathol, 60, 709-14.

Shahabuddin MD, RaghuveerCV (2003). Reed-Sternberg-like cells in T-cell rich B-cell lymphoma: a diagnostic dilemma. Indian J Pathol Microbiol, 46, 55-6.

Sigvardsson M, Clark DR, Fitzsimmons D, et al (2002). Early B-cell factor E2A and Pax-5 co operate to activate the early B cell-specific mb-1 promoter. Mol Cell Biol, 22, 8539-51.

Sultan S, Irfan SM, Parveen S, Ali S (2016).Clinico-Hematological findings for classical Hodgkin's Lymphoma: an institutional experience. Asian Pac J Cancer Prev, 17, 4009-11

Swerdlow SH, Campo E, Harris NL, et al (2008). WHO classification of tumours of haematopoietic and lymphoid tissues. International Agency for Research on Cancer (IARC).

ZwolloZwollo P, Arrieta H, Ede K, et al (1997).The Pax-5 gene is alternatively spliced during B-cell development. $J$ Biol Chem, 272, 10160-8.

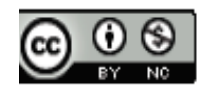

This work is licensed under a Creative Commons AttributionNon Commercial 4.0 International License. 